\title{
CIGB-552, a promising candidate to cancer therapy.
}

\author{
Brizaida Oliva Arguelles ${ }^{1}$, Mario Riera-Romo ${ }^{2}$, and Maribel Guerra Vallespi ${ }^{1}$ \\ ${ }^{1}$ Center for Genetic Engineering and Biotechnology \\ ${ }^{2}$ Institute of Marine Sciences
}

May 5, 2020

\begin{abstract}
Peptide-based cancer therapy has been of great interest due to the unique advantages of peptides, such as the low molecular weight, the ability to specifically target tumor cells, easy availability and low toxicity in normal tissues. Therefore, identify and synthesize novel peptides could provide a promising choice to patients with cancer. The antitumor second generation peptide CIGB-552 has been developed as a candidate to cancer treatment. Proteomic and genomic studies have identified the intracellular protein COMMD1 as the specific target of CIGB-552. This peptide penetrates inside tumor cells to induce the proteasomal degradation of RelA, causing the termination of NF-kB signaling. The antitumor activity of CIGB-552 has been validated in vitro in different human cancer cell lines, as well as in vivo in syngeneic and xenograft tumor mouse models and in cancer-bearing dogs. The aim of this review is to present and discuss the experimental data about CIGB-552, its mechanism of action and its therapeutic potential in human chronic diseases. This peptide is already in phase I of clinical trials as antineoplastic drug, but also has possible application to other inflammatory and metabolic conditions.
\end{abstract}

\section{Introduction}

Cancer is a multifactorial and complex disease, which presents different clinical outcomes depending on the affected tissue and the genetic background of the patient (Luo, Solimini, \& Elledge, 2009). Tumor cells express a variety of molecular targets involved in cancer progression and exhibit a deregulation in normal growth, proliferation and survival, among other vital functions (Hanahan \& Weinberg, 2011). Because of its complexity, the huge variety of molecular targets involved and the high variability in therapeutic response, cancer has become a health problem worldwide and one the sickness most difficult to treat in the 21st century.

Commonly, traditional chemotherapeutical drugs target tumor cells by disrupting necessary cell products, such as DNA, RNA, or proteins (Huang et al., 2014). However, chemotherapy is also insufficient and highly toxic, because it does not specifically target tumor cells, causing many side effects in patients (Amit \& Hochberg, 2010). Additionally, multidrug resistance (MDR) is the main reason by which chemotherapy fails to cure patients (Huang et al., 2014). Under these limitations, therapeutical strategies based on peptides are receiving increased attention.

There are several advantages of peptides, such as the small size, easy synthesis and modification, tumor penetrating ability and a good biocompatibility (Wu et al., 2014). A growing number of studies indicate that peptides may be beneficial for drug discovery and development. Peptides offer minimal immunogenicity, excellent tissue penetrability, low-cost manufacturability, and relatively easy of modify to enhance in vivo stability and biological activity, properties which make them ideal candidates for cancer treatment (Yavari, Mahjub, Saidijam, Raigani, \& Soleimani, 2018).

Peptides have also demonstrated to play a role in cancer therapy, including early diagnosis, prognostic predictors, and directly in the treatment of cancer patients. Unlike other therapies, peptides seem to be more effective due to their specificity. Recently, some peptide-based treatments against cancer, such as 
peptide vaccines, have attracted increased attention. Anticancer activity of different peptides is attributed to a variety of mechanisms that restrict tumor growth. (Borghouts, Kunz, \& Groner, 2005).

CIGB-552 is a synthetic peptide "first-in-class" that increases the level of the intracellular protein COMMD1 (Copper Metabolism Mur 1 Domain containing protein1) and inhibits the anti-apoptotic genes regulated by Nuclear Factor $\chi \mathrm{B}(\mathrm{NF}-\varkappa \mathrm{B})$. CIGB-552 leads to the selective degradation of RelA, an NF- $\chi \mathrm{B}$ subunit, and induces apoptosis in multiple types of tumor cells in the absence of toxicity to normal cells. In addition, CIGB-552 inhibits the transcriptional activity of NF- $\chi \mathrm{B}$ induced by TNF- $\alpha$ and IL- $1 \beta$ in human colon cancer cells. The mechanism of action of CIGB-552 assumes a new-targeted anticancer therapy to regulate oncogenic-inflammatory activity of NF- $x \mathrm{~B}$ in cancer cells, providing selectivity and specificity. This novel peptide has a potential application against solid tumors and inflammation-associated cancer including colorectal, breast and lung cancer, lymphomas and others.

\section{Origin of CIGB-552 peptide}

In the Centre for Genetic Engineering and Biotechnology (CIGB), Havana, Cuba, Vallespi and colleagues obtained an antimicrobial peptide against septic shock designed from the region 31-52 of the Limulusanti-LPS factor (LALF), a protein derived from the Horseshoe crabLimulus polyfemus (Vallespi, Colas, Garay, Reyes, \& Arana, 2004). The peptide CIGB-550 (LALF $31-52$ ) demonstrated cell penetrating capacity due to net positive charge and amphipathic structure by alternating positive/ hydrophobic basic residues. It showed the capacity to bind, neutralize bacterial lipopolisacharide (LPS) and block the inflammatory response mediated by this molecule. Vallespi et al. demonstrated anti-inflammatory and immunomodulatory properties for CIGB-550, but also showed an antiviral effect for this peptide, mediated by IFN- $\gamma$ and IFN- $\alpha$ (Vallespi et al., 2003). It has been reported that antimicrobial peptides exhibit a broad spectrum of cytotoxic activity against cancer cells (Hoskin \& Ramamoorthy, 2008) and CIGB-550 is no an exception. The ability to inhibit processes associated with heparin, such as anticoagulation, angiogenesis, and tumor cell proliferation, was attributed to this peptide, although there are no experimental data supporting this. In order to study the structurefunction relationship of $\mathrm{LALF}_{31-52}$ and its connection with the biological properties attributed to the peptide, a synthetic library was generated by alanine scanning (Vallespi et al., 2010). The resulting peptides were evaluated by LPS binding ability, antitumor effect, penetration capacity to live cells and immunostimulatory activity. These results lead to the development of L-2, a peptide optimized for anticancer activity. L-2 has lost its LPS binding capacity, however is a powerful cytotoxic agent against murine and human tumor cell lines (Vallespi et al., 2010). One of the weaknesses of the peptides as drugs is the short half-life and fast elimination (Fosgerau \& Hoffmann, 2015). By modifications in the primary structure of L-2, we have developed a second-generation peptide as anticancer drug for evaluating in clinical trials named CIGB-552 (Figure 1). This peptide maintains the cell penetrating capacity and shows a higher antitumor effect in comparison to CIGB-550 and L-2.

\section{CIGB-552 has antitumor effects in vitro and in vivo}

The studies of anticancer activity of CIGB-552 were performed usingin vitro culture systems as well as clinically relevant in vivo models, as is shown in figure 2 . We began with the demonstration of a selective antiproliferative activity in several cancer cell lines. We demonstrated that CIGB-552 has powerful antiproliferative and cytotoxic effects compared to its precursor peptide L-2. In this assays, we evaluated the effect of the peptide on non-tumor cell lines where the $\mathrm{IC}_{50}$ was higher respect to tumor cells. These differences suggest the selectivity of this peptide to malignant cells. The $\mathrm{IC}_{50}$ values observed in tumor cell lines suggest a tumor type-dependent pattern of sensitivity (Fernandez Masso et al., 2013).

Since apoptosis often occurs as a consequence of a cell cycle blockade, we checked whether the cytotoxicity of L-2 was mediated by an alteration of the cell division cycle. Analyses by flow cytometry of tumor cells treated with L-2, showed the absence of the G2/M peak and the accumulation of cells in S phase. Recently, we demonstrated that this difference is caused by the internalization mechanism of the peptide (Vallespi et al., 2010).

The in vivo therapy on solid tumors was a subsequent phase in the proof of concept of CIGB-552 as an 
anticancer drug (Figure 2). In our institute, we developed a murine model of tumor CT-26. This model was used to study the administration route and the dosage regimen for the treatment. Firstly, we demonstrated that the subcutaneous administration of CIGB-552 led to a significant reduction of tumor growth compared to the group treated with saline solution. After two week of treatment, the tumor volume was decreased and this behavior was observed until the end of the study (Vallespi et al., 2014). Therefore, this in vivo study demonstrated that subcutaneous administration of CIGB- 552 was able to inhibit the $50 \%$ of tumor growth injecting the peptide once a week, during 2 weeks. In collaboration with an EPO-Berlin institute, we further evaluated the effect of a subcutaneous administration of CIGB-552 in a xenograft model of human colon cancer HT-29. In this mouse model, we analyzed the body weight as an indicator of tolerability. As occurs in the syngeneic CT26 model, the peptide showed a significant reduction of tumor volume compared with the control group. All mice of groups treated with CIGB-552 keep the body weight during the study, indicating safety and tolerability. This study included a group treated with Oxaliplatin, a standard drug in the therapy of colorectal cancer. Oxaliplatin induced a significant inhibition of tumor growth, but this effect was accompanied by a severe loss of body weight and two deaths probably due to its high toxicity (Vallespi et al., 2014).

The mechanisms involved in the reduction of tumor growth in response to CIGB-552 were evaluated in both murine syngeneic and human xenograft models. In vivo, the groups treated with CIGB-552 exhibit induction of apoptosis in the tumor and a decrease of microvessels density (Vallespi et al., 2014). The resistance to programed cell death and the sustained angiogenesis are included in a group of properties called hallmarks of cancer, a set of functional capabilities acquired during the multistep development of human tumors. Thus, the antitumor mechanism of the peptide CIGB-552 is associated with proteins and signaling pathways that trigger apoptosis inhibit the proliferation and induce the reduction of blood vessels formation.

Monolayers of tumor cells cultivated in vitro and mouse xenografts implanted with those cells, have been the standard toolkit to cancer biologists for decades. However, the need for better and more clinically predictive models of human cancer is imminent. Spontaneous cancer in pet dogs is considered an attractive model to study the efficacy of drug candidates to cancer therapy (Rowell, McCarthy, \& Alvarez, 2011). Cancers in affective animals such as pet dogs are characterized by histological features similar to human cancer, tumor growth over long periods, inter-individual and intra-tumor heterogeneity, the development of recurrent or resistant diseases, and metastasis to relevant distal sites (Hawai et al., 2013). Based on that, we evaluated the safety, tolerability and antineoplastic effect of CIGB-552 in tumor-bearing dogs. In this study, our group keeps the same dosage regimen used by subcutaneous route in mouse models. The treatment with CIGB-552 had not any negative effect on total leukocytes, do not affect the levels of liver transaminases or hemoglobin during the study (Vallespi et al., 2017). Interesting, the white blood count return to normal levels after completing the study, which suggest that treatment with the peptide do not induce leucopenia, a common issue in chemotherapy regimens. The dogs enrolled into the study showed a tumor regression (> $50 \%$ ), demonstrating a stabilization of the disease (Vallespi et al., 2017).

Altogether, these preclinical results validate the peptide CIGB-552 as a potent antiproliferative and antitumor drug which mechanism is related with the inhibition of various hallmarks of cancer. In addition, the subcutaneous administration of CIGB-552 is safe and successful as an anticancer treatment at least, at preclinical level.

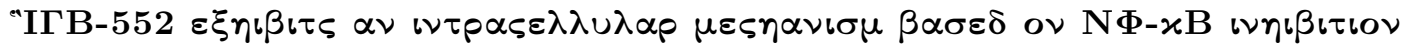

\section{Cell penetrating Capacity}

The cellular uptake mechanism of cell penetrating peptides (CPPs) is an essential piece of optimization of appropriate strategies for in vivo therapeutic applications. CPPs have been described to enter cells either by the classical endocytic pathway or through an energy-independent mechanism referred to as transduction (Heitz, Morris, \& Divita, 2009). In spite of the requirements needed for each mechanism, different internalization patterns are also observed inside the cells. Endocytosis produces a punctuated pattern consequence of peptide internalization into endocytic vesicles (Ter-Avetisyan et al., 2009). Meanwhile, transduction by 
direct membrane penetration results in fully loaded cells displaying a homogeneous distribution throughout the cytoplasm and nucleoplasm (Tunnemann et al., 2008).

The precursor peptides CIGB-550 and L-2 have the capacity to penetrate the cell, so we studied the internalization kinetic of CIGB-552 after modifications in its primary structure. Both endocytosis and transduction are involved in CIGB-552 internalization in the three cell lines evaluated, MCF-7 (human breast adenocarcinoma), NCI-H460 (human large cell lung cancer carcinoma) and HT-29 (human colorectal adenocarcinoma). We analyzed the internalization of fluorescent labeled CIGB-552 in these cell lines by confocal microscopy. The intracellular uptake of the peptide was observed in the cytoplasm after 10 minutes and shows mainly punctuate staining close to the nuclei (Fernandez Masso et al., 2013; Vallespi et al., 2014). Expression of two different proteins Rab5A and Fibrillarin were analyzed. Rab5A is a small GTPase, which has been found to be expressed in early endosomes (Bucci et al., 1992), whereas Fibrillarin, which possesses methyltransferase activity, is a commonly used marker of active nucleoli (Rodriguez-Corona, Sobol, Rodriguez-Zapata, Hozak, \& Castano, 2015). Therefore, the use of these two proteins, allowed us to define CIGB-552 localization with respect to both endosomes and nucleoli (Figure 3). Co-localization was found among CIGB-552 and these two markers, indicating that this peptide is effectively able to enter cells through different mechanisms (Astrada et al., 2016).

However, CIGB-552 incorporation efficiency and contribution of each mechanism is cell-line dependent. Astrada et al. showed that cell-penetrating capacity varies among the three cell lines studied. H460 displayed the highest internalization levels, while MCF-7 presented the lowest internalization capacity. Moreover, CIGB-552 used both endocytosis and transduction as internalization mechanisms, although the contributions of each mechanism varied among the studied cell lines (Astrada, Fernandez Masso, Vallespi, \& BollatiFogolin, 2018). Altogether, our results suggest that NCI-H460 sensitivity could be explained by its high internalization capacity of CIGB-552, by using endocytosis as a preferred mechanism, which in turn could facilitate the interaction between the peptide and target proteins resident in the endosomal compartment. Endocytosis constitutes the most effective way of transporting the CIGB-552 peptide inside cells, thus promoting higher sensitivity towards peptide cytotoxic effects.

Biological processes involved in the antitumor effect of CIGB-552

Since CIGB-552 is a synthetic peptide that possess modified aminoacids (D aminoacids), which cannot be translated inside cells, we decided to use L-2 peptide instead, for gene expression studies. L-2 represents the primary sequence that has been modified in order to generate a more stable peptide, the CIGB- 552 . Subtractive hybridization (SSH) analysis on laryngeal tumor Hep-2 cells showed that L-2 peptide treatment impacts the expression of genes related to biological processes and pathways involved in cancer such as: DNA repair, mitosis, and angiogenesis (Vallespi et al., 2010). Furthermore, a yeast two-hybrid study of L-2 and a pull-down technique identified COMMD1 protein as a target of CIGB-552. The results of two hybrid and pull-down experiments indicate that the interaction between peptides and COMMD1 is specific and the strength of this interaction may be relevant for the antitumor effect of the peptides (Fernandez Masso et al., 2013). This interaction was recently confirmed in live cells measuring the in situ COMMD1 expression level after treatment with CIGB-552 (Astrada et al., 2018). We found that in MCF-7 and NCIH460 cells the peptide accumulated COMMD1 in the cytoplasm. In addition, we identified the interaction between CIGB-552 and COMMD1 as a co-localization of both proteins at early endosome using confocal microscopy approach. This result was corroborated by ELISA assay (Astrada et al., 2016). Such finding is very interesting to the mechanism of CIGB-552, because define a specific intracellular target for the peptide and the initial cellular compartment were this interaction occurs.

To identify other proteins that interact with CIGB-552, two chemical proteomic approaches were then conducted using PBS-soluble proteins derived from Hep-2 cells (Rodriguez-Ulloa et al., 2015). A total of 161 proteins constitute the identified potential target profile of CIGB-552. Biological processes related to carbohydrate metabolism, protein modification, and cell cycle, are significantly represented on this dataset. Interestingly, such biological processes are also represented in the transcriptomic profile regulated by L-2 peptide in Hep-2 tumor cells. Functional subnetworks which are perturbed by CIGB-552 include anti- 
apoptosis and negative regulation of cell cycle; extracellular structure organization and response to hypoxia. Positive regulation of NF-?B transcription factor activity is disrupted by the CIGB-552 target profile essentially at two network nodes: RelA and TRAF6 (Rodriguez-Ulloa et al., 2015). On the other hand, we study the proteins modulated by treatment with CIGB-552 in HT-29 cells using subcellular protein and peptide fractionation by chemical proteomic approach. In particular, we explored the nuclear proteome of HT-29 cells at five hours of treatment with CIGB-552, identifying 68 differentially modulated proteins, 49 of which localize to the nucleus (Nunez de Villavicencio-Diaz et al., 2015). The differentially modulated proteins were analyzed following a system biology approach. Results pointed to a modulation of apoptosis, oxidative stress, $\mathrm{NF}-x \mathrm{~B}$ activation, inflammatory signaling and cell adhesion and motility. These results demonstrated that even in different cell lines (HT-29, Hep-2) the CIGB-552 antitumor effect is exerted by modulating similar biological pathways.

According to proteomic and genomic data, oxidative stress and apoptosis are the main biological processes modulated by CIGB-552 in tumor cells (Figure 3). This makes sense, considering the crucial role of COMMD1 in both cellular functions. COMMD1 impairs the antioxidant superoxide dismutase 1 (SOD1) activity by reducing the expression levels of active SOD1 homodimers, late in the posttranslational maturation process of this enzyme (Vonk, Wijmenga, Berger, van de Sluis, \& Klomp, 2010). Furthermore, COMMD1 is also involved in apoptotic cell death, mainly due to negative regulation of NF- $x \mathrm{~B}$ (Thoms et al., 2010). Consequently, the treatment with CIGB-552 must be able to induce oxidative damage and apoptosis in cancer cells. In fact, this was demonstrated in human lung cancer cells in presence of the peptide, which increased the levels of protein and lipid peroxidation as a sign of oxidative stress damage (Fernandez Masso et al., 2013). In addition, the downregulation of COMMD1 in these cells abrogated the negative effect of CIGB-552 on SOD1 activity, demonstrating the contribution of COMMD1 to this process. In the same way, the peptide activated the apoptotic pathway in human lung cancer cells trough the modulation of the Bax/Bcl-2 protein ratio and the cleavage of caspase 3 and PARP (Fernandez Masso et al., 2013).

COMMD1 and NF- $\varkappa$ B activity

The COMMD protein family is highly conserved among multicellular eukaryotic organisms. COMMD1 is the best characterized member of the family and is conserved among vertebrates (Burstein et al., 2005). This protein represents a pleiotropic factor involved in the regulation of many cellular and physiological processes that include oxidative stress, protein aggregation, protein trafficking, NF- $x$ B-mediated transcription and oncogenesis (Bartuzi et al., 2016; Phillips-Krawczak et al., 2015; Vonk et al., 2010). The potential of COMMD1 in cancer therapy is becoming a focus of attention and this protein offers a way to modulate crucial events in oncogenesis and even produces ROS that contribute with apoptosis of tumor cells, in a safe and specific manner (Riera-Romo, 2018).

The proteomics and genomics approach indicated that COMMD1 represents a molecular target of the peptide CIGB-552. The development of the interactome of CIGB-552, where the data obtained by genomics and proteomics study was integrated, suggests a direct connection with the NF- $x \mathrm{~B}$ pathway, in a COMMD1dependent manner. According to these findings, the cellular expression of COMMD1 in whole-cell lysates of human cancer cells of different histological origin was determined using Western blot analysis. These experiments revealed an increase in the levels of endogenous COMMD1 after five hours of treatment with the peptide (Fernandez Masso et al., 2013). This effect on COMMD1 was not accompanied by significant changes in mRNA expression of the protein, suggesting a posttranscriptional effect of CIGB-552 on COMMD1 levels (Fernandez Masso et al., 2013). It is known that nuclear localization of the protein COMMD1 accelerates the ubiquitination and degradation of the RelA subunit of NF- $\chi \mathrm{B}$ and decreases the activation of antiapoptotic genes (Maine, Mao, Komarck, \& Burstein, 2007; Thoms et al., 2010). In this sense, we demonstrated that in response to CIGB-552, COMMD1 localizes into the nucleus, a fact that is related with increasing amounts of ubiquitinated RelA and apoptosis induction (Figure 3). This effect was abrogated decreasing the levels of COMMD1 by interferent RNA (iRNA) gene silencing method (knockdown), indicating the functional role of this protein in the antitumor activity of CIGB-552 (Fernandez Masso et al., 2013).

As a downstream event, the transcriptional activity of NF- $\varkappa \mathrm{B}$ was evaluated in the reporter cell line HT-29- 
NF- $x$ B-hrGFP E5. The peptide CIGB- 552 inhibits the NF- $x$ B activity in these cells in presence or absence of proinflammatory cytokines such as TNF- $\alpha$ and IL-1 $\beta$. Besides, CIGB-552 reduces the levels of IL-8 in cell culture supernatants confirming the inhibition of NF- $\chi \mathrm{B}$ activity (Nunez de Villavicencio-Diaz et al., 2015).

\section{Role of the primary structure in the antitumor mechanism of CIGB-552.}

Biostability of peptides in blood and serum is an important issue for development of peptides as clinically drugs. In vitro degradation of peptides in serum and plasma is considered the primary methodology for studying the degradation pattern of peptides (Werle, 2006). For that reason, our group examined the in vitro metabolic stability of CIGB-552 in serum during 120 minutes. A typical serine-proteases degradation pattern was suggested for this peptide sequence (Vallespi et al., 2014). The main metabolites of CIGB552 showed no great differences regarding hydrophobicity, hydrophilicity, and isoelectric point. However, the analysis of the cytotoxic effect, cell-penetrating capacity, antitumor mechanism and interaction with its intracellular target, COMMD1, demonstrated a loss of antitumor capacity for the metabolites compared to CIGB-552 (Figure 4). These findings suggest the importance of C-terminal amino acidic residues (Lys18, Phe19 and Trp20) in the antitumor activity of CIGB-552 (Astrada et al., 2016). The role of these residues in the peptide-membrane interaction was analyzed by molecular dynamic simulation (Astrada et al., 2016). CIGB-552 and the 17 amino acid metabolite (named as 5) have a conserved structural motif. Two tryptophan residues and a tyrosine create a hydrophobic cluster that brings together the C-terminal carboxylate moieties and one arginine residue, forming a stable salt bridge interaction that leads to a looped conformation. Indeed, tryptophan-tryptophan and arginine-C-terminal carboxylate distances in both systems display very stable interactions.

Altogether, these results demonstrate that the primary structure of CIGB- 552 is considered the minimum active sequence needed to produce a potent antitumor effect in cell and animal models. In this sense, the protection of CIGB-552 from proteolitic degradation is one of the most important challenges that face this peptide in its development as an anticancer drug. The use of different encapsulation systems such as liposomes, multilayer emulsions or nanoparticle vehicles could be an alternative to deal with this issue.

\section{Conclusions and future perspectives}

CIGB-552 is a "first-in class" antitumor peptide developed by a series of modifications from a structural motif present in the antimicrobial protein LALF. This peptide in capable of penetrate inside the cell to interact with different target proteins among which COMMD1 has been identified. This pleotropic protein regulates the stability and activity of several intracellular, endosomal and transmembrane proteins, regulating a variety of biological processes. It has been described to participate in the termination of NF- $\varkappa \mathrm{B}$ signalling and in the negative regulation of SOD1, among other molecular events. CIGB-552 acting through COMMD1, is able of induce oxidative stress and trigger apoptosis in cancer cells and is effective, safe an tolerable in different animal models of cancer including mice and dogs, what makes it a promising candidate to cancer therapy. In fact this peptide drug is already in clinical trials and has concluded recently the phase I study, which demonstrated an overall positive effect in patients with no toxicity or side effects.

In spite of that, the potential of CIGB-552 is still under evaluation. Recent studies have revealed new findings about COMMD1 that could expand the therapeutic applications of this peptide. Fedoseienko et al . demonstrated that nuclear COMMD1 decreased protein expression of the DNA repair gene BRCA1 and the apoptosis inhibitor BCL2 in ovarian cells A-2780, conferring sensitivity to Cisplatin (Fedoseienko et al., 2016). In addition, they demonstrated through tissue micro-arrays that nuclear expression of COMMD1 is associated with an improved response to chemotherapy. This information supports the idea of the use of CIGB-552 in combined therapy with other chemotherapeutic drugs, to improve antitumor effect and reduce toxicity. In the same way, Yeh et al examined the association of COMMD1 with stemness. The downregulation of COMMD1 amplifies stemness-associated property of cancer cells (Yeh et al., 2016), suggesting that CIGB552 could target cancer steam cells, which are involved in tumor re-emergence, metastasis and multidrug resistance. Furthermore, COMMD1 has been reported as a negative regulator of the hypoxia inducible factor 1 (HIF-1), which is implicated in tumor angiogenesis and metastasis (van de Sluis et al., 2009; van de Sluis 
et al., 2010), thus, this could be another cancer-associated process modulated by CIGB-552. Interestingly, in accordance with this hypothesis the peptide reduced microvessel density in vivo in our CT-26 tumor mouse model.

CIGB-552 differentially modulates genes and proteins involved in key signaling pathways of cancer cells such as NF $\varkappa$ B, hypoxia, apoptosis and inflammation. The recovery of COMMD1 levels by CIGB-552 negatively regulates the $\mathrm{NF} x \mathrm{~B}$ activity in absence or presence of inflammatory cytokines. Therefore, the molecular mechanism described for CIGB-552 validate its pharmacological use in chronic diseases such as human cancer, by inhibiting inflammation, tumor angiogenesis and stemness process or even in certain inflammatory conditions such as colitis and colitis-related cancer, Inflammatory Bowel Disease, Crohn's Disease among others. Furthermore, CIGB-552 could be used in combination regimens with non-steroidal anti-inflammatory drugs (NSAID), antineoplastic agents and other target therapies. Substantial evidence indicates that aspirin and related NSAIDs have potential as chemopreventive/therapeutic agents (Drew, Cao, \& Chan, 2016; Patrignani \& Patrono, 2016). However, these drugs cannot be universally recommended for prevention purposes due to their potential side-effect profiles. Stark et al have demonstrated that Aspirin inhibits the transcriptional activity of NF $\varkappa B$ through stabilization of COMMD1 in colon cancer cells (O'Hara et al., 2014; Thoms et al., 2010). The intervention of CIGB-552 in combination with Aspirin could mediate an improved effect and could reduce the clinical dose of both drugs in cancer therapy. The constitutive activation of $\mathrm{NF}-x \mathrm{~B}$ is one of the resistance mechanisms to antineoplastic treatments. The inhibition of NF- $x \mathrm{~B}$ activity by CIGB-552 could be an attractive strategy to sensitize tumors to antineoplastic agents, reducing the doses of drugs and providing a better life quality for patients.

Increasing evidence is pointing out COMMD1 as an attractive molecular target involved in a variety of human disorders. CIGB-552 as a biotechnological product that accumulate this protein and potentiate its intracellular effect, represent an interesting candidate not only for cancer therapy, but also to other metabolic diseases in which COMMD1 is implicated. For example, the research group of Fedoseienko et al. has also studied the role of COMMD1 in atherosclerosis. The localization of COMMD1 in the endosome is critical for Low Density Lipoprotein Receptor (LDLR) recycling and cholesterol levels in mice (Bartuzi et al., 2016). Likewise, Drevillon et al demonstrate that COMMD1 modulates the activity and surface expression of the Cystic Fibrosis Transmembrane Conductance Regulator (CFTR) in epithelial cells, an activity that is down-regulated in cystic fibrosis patients (Drevillon et al., 2011). In line with this, CIGB-552 could be a therapeutical alternative to atherosclerosis and cystic fibrosis, two human disorders that do not possess a current effective treatment. The molecular evidence and experimental data discussed throughout this review reveal the peptide CIGB-552 as an interesting, effective and versatile drug that could be developed as a novel anti-inflammatory and anticancer drug, which could be effective for different human chronic conditions.

\section{References}

Amit, D., \& Hochberg, A. (2010). Development of targeted therapy for bladder cancer mediated by a double promoter plasmid expressing diphtheria toxin under the control of H19 and IGF2-P4 regulatory sequences. $J$ Transl Med, 8 , 134. doi: 10.1186/1479-5876-8-134

Astrada, S., Fernandez Masso, J. R., Vallespi, M. G., \& Bollati-Fogolin, M. (2018). Cell Penetrating Capacity and Internalization Mechanisms Used by the Synthetic Peptide CIGB-552 and Its Relationship with Tumor Cell Line Sensitivity.Molecules, 23 (4). doi: 10.3390/molecules23040801

Astrada, S., Gomez, Y., Barrera, E., Obal, G., Pritsch, O., Pantano, S., . . . Bollati-Fogolin, M. (2016). Comparative analysis reveals amino acids critical for anticancer activity of peptide CIGB-552. J Pept Sci, 22 (11-12), 711-722. doi: 10.1002/psc.2934

Bartuzi, P., Billadeau, D. D., Favier, R., Rong, S., Dekker, D., Fedoseienko, A., . . . van de Sluis, B. (2016). CCC- and WASH-mediated endosomal sorting of LDLR is required for normal clearance of circulating LDL. Nat Commun, 7 , 10961. doi: 10.1038/ncomms10961

Borghouts, C., Kunz, C., \& Groner, B. (2005). Current strategies for the development of peptide-based 
anti-cancer therapeutics. J Pept Sci, 11 (11), 713-726. doi: 10.1002/psc.717

Bucci, C., Parton, R. G., Mather, I. H., Stunnenberg, H., Simons, K., Hoflack, B., \& Zerial, M. (1992). The small GTPase rab5 functions as a regulatory factor in the early endocytic pathway. Cell, 70 (5), 715-728. doi: 10.1016/0092-8674(92)90306-w

Burstein, E., Hoberg, J. E., Wilkinson, A. S., Rumble, J. M., Csomos, R. A., Komarck, C. M., . . . Duckett, C. S. (2005). COMMD proteins, a novel family of structural and functional homologs of MURR1. J Biol Chem, 280 (23), 22222-22232. doi: 10.1074/jbc.M501928200

Drevillon, L., Tanguy, G., Hinzpeter, A., Arous, N., de Becdelievre, A., Aissat, A., . . . Fanen, P. (2011). COMMD1-mediated ubiquitination regulates CFTR trafficking. PLoS One, 6 (3), e18334. doi: 10.1371/journal.pone.0018334

Drew, D. A., Cao, Y., \& Chan, A. T. (2016). Aspirin and colorectal cancer: the promise of precision chemoprevention. Nat Rev Cancer, 16 (3), 173-186. doi: 10.1038/nrc.2016.4

Fedoseienko, A., Wieringa, H. W., Wisman, G. B., Duiker, E., Reyners, A. K., Hofker, M. H., . . . van Vugt, M. A. (2016). Nuclear COMMD1 Is Associated with Cisplatin Sensitivity in Ovarian Cancer. PLoS One, 11 (10), e0165385. doi: 10.1371/journal.pone.0165385

Fernandez Masso, J. R., Oliva Arguelles, B., Tejeda, Y., Astrada, S., Garay, H., Reyes, O., . . . Vallespi, M. G. (2013). The Antitumor Peptide CIGB-552 Increases COMMD1 and Inhibits Growth of Human Lung Cancer Cells. J Amino Acids, 2013 , 251398. doi: 10.1155/2013/251398

Fosgerau, K., \& Hoffmann, T. (2015). Peptide therapeutics: current status and future directions. Drug Discov Today, 20 (1), 122-128. doi: 10.1016/j.drudis.2014.10.003

Hanahan, D., \& Weinberg, R. A. (2011). Hallmarks of cancer: the next generation. Cell, 144 (5), 646-674. doi: 10.1016/j.cell.2011.02.013

Hawai, S. M., Al-Zayer, M., Ali, M. M., Niu, Y., Alawad, A., Aljofan, M., . . . Altuwaijri, S. (2013). Dogs: active role model for cancer studies - a review.

Heitz, F., Morris, M. C., \& Divita, G. (2009). Twenty years of cell-penetrating peptides: from molecular mechanisms to therapeutics. Br J Pharmacol, 157 (2), 195-206. doi: 10.1111/j.1476-5381.2009.00057.x

Hoskin, D. W., \& Ramamoorthy, A. (2008). Studies on anticancer activities of antimicrobial peptides.Biochim Biophys Acta, 1778 (2), 357-375. doi: 10.1016/j.bbamem.2007.11.008

Huang, W., Seo, J., Willingham, S. B., Czyzewski, A. M., Gonzalgo, M. L., Weissman, I. L., \& Barron, A. E. (2014). Learning from host-defense peptides: cationic, amphipathic peptoids with potent anticancer activity. PLoS One, 9 (2), e90397. doi: 10.1371/journal.pone.0090397

Luo, J., Solimini, N. L., \& Elledge, S. J. (2009). Principles of cancer therapy: oncogene and non-oncogene addiction. Cell, 136 (5), 823-837. doi: 10.1016/j.cell.2009.02.024

Maine, G. N., Mao, X., Komarck, C. M., \& Burstein, E. (2007). COMMD1 promotes the ubiquitination of NF-kappaB subunits through a cullin-containing ubiquitin ligase.EMBO J, 26 (2), 436-447. doi: 10.1038/sj.emboj.7601489

Nunez de Villavicencio-Diaz, T., Ramos Gomez, Y., Oliva Arguelles, B., Fernandez Masso, J. R., RodriguezUlloa, A., Cruz Garcia, Y., . . . Guerra Vallespi, M. (2015). Comparative proteomics analysis of the antitumor effect of CIGB-552 peptide in HT-29 colon adenocarcinoma cells. J Proteomics, 126 , 163-171. doi: $10.1016 /$ j.jprot.2015.05.024

O'Hara, A., Simpson, J., Morin, P., Loveridge, C. J., Williams, A. C., Novo, S. M., \& Stark, L. A. (2014). p300-mediated acetylation of COMMD1 regulates its stability, and the ubiquitylation and nucleolar translocation of the RelA NF-kappaB subunit. J Cell Sci, 127 (Pt 17), 3659-3665. doi: 10.1242/jcs.149328 
Patrignani, P., \& Patrono, C. (2016). Aspirin and Cancer. J Am Coll Cardiol, 68 (9), 967-976. doi: 10.1016/j.jacc.2016.05.083

Phillips-Krawczak, C. A., Singla, A., Starokadomskyy, P., Deng, Z., Osborne, D. G., Li, H., . . . Burstein, E. (2015). COMMD1 is linked to the WASH complex and regulates endosomal trafficking of the copper transporter ATP7A. Mol Biol Cell, 26 (1), 91-103. doi: 10.1091/mbc.E14-06-1073

Riera-Romo, M. (2018). COMMD1: A Multifunctional Regulatory Protein. J Cell Biochem, 119 (1), 34-51. doi: $10.1002 /$ jcb.26151

Rodriguez-Corona, U., Sobol, M., Rodriguez-Zapata, L. C., Hozak, P., \& Castano, E. (2015). Fibrillarin from Archaea to human. Biol Cell, 107 (6), 159-174. doi: 10.1111/boc.201400077

Rodriguez-Ulloa, A., Gil, J., Ramos, Y., Hernandez-Alvarez, L., Flores, L., Oliva, B., . . . Guerra-Vallespi, M. (2015). Proteomic Study to Survey the CIGB-552 Antitumor Effect.Biomed Res Int, 2015 , 124082. doi: $10.1155 / 2015 / 124082$

Rowell, J. L., McCarthy, D. O., \& Alvarez, C. E. (2011). Dog models of naturally occurring cancer.Trends Mol Med, 17 (7), 380-388. doi: 10.1016/j.molmed.2011.02.004

Ter-Avetisyan, G., Tunnemann, G., Nowak, D., Nitschke, M., Herrmann, A., Drab, M., \& Cardoso, M. C. (2009). Cell entry of arginine-rich peptides is independent of endocytosis. J Biol Chem, 284 (6), 3370-3378. doi: $10.1074 / \mathrm{jbc} . \mathrm{M} 805550200$

Thoms, H. C., Loveridge, C. J., Simpson, J., Clipson, A., Reinhardt, K., Dunlop, M. G., \& Stark, L. A. (2010). Nucleolar targeting of RelA(p65) is regulated by COMMD1-dependent ubiquitination. Cancer Res, 70 (1), 139-149. doi: 10.1158/0008-5472.CAN-09-1397

Tunnemann, G., Ter-Avetisyan, G., Martin, R. M., Stockl, M., Herrmann, A., \& Cardoso, M. C. (2008). Live-cell analysis of cell penetration ability and toxicity of oligo-arginines. J Pept Sci, 14 (4), 469-476. doi: $10.1002 /$ psc. 968

Vallespi, M. G., Alvarez-Obregon, J. C., Rodriguez-Alonso, I., Montero, T., Garay, H., Reyes, O., \& Arana, M. J. (2003). A Limulus anti-LPS factor-derived peptide modulates cytokine gene expression and promotes resolution of bacterial acute infection in mice. Int Immunopharmacol, 3 (2), 247-256. doi: 10.1016/S1567$5769(02) 00277-1$

Vallespi, M. G., Colas, M., Garay, H., Reyes, O., \& Arana, M. J. (2004). Differential regulation of Th1/Th2 in relevant tissues for sepsis pathogenesis with a Limulus anti-LPS factor-derived peptide increases survival in Gram-positive sepsis. Int Immunopharmacol, 4 (10-11), 1343-1351. doi: 10.1016/j.intimp.2004.05.019

Vallespi, M. G., Fernandez, J. R., Torrens, I., Garcia, I., Garay, H., Mendoza, O., . . . Reyes, O. (2010). Identification of a novel antitumor peptide based on the screening of an Ala-library derived from the LALF(32-51) region. J Pept Sci, 16 (1), 40-47. doi: 10.1002/psc.1192

Vallespi, M. G., Pimentel, G., Cabrales-Rico, A., Garza, J., Oliva, B., Mendoza, O., . . . Reyes, O. (2014). Antitumor efficacy, pharmacokinetic and biodistribution studies of the anticancer peptide CIGB-552 in mouse models. J Pept Sci, 20 (11), 850-859. doi: 10.1002/psc.2676

Vallespi, M. G., Rodriguez, J. C., Seoane, L. C., Alvarez, P., Santana, H., Garay, H., . . . Reyes, O. (2017). The first report of cases of pet dogs with naturally occurring cancer treated with the antitumor peptide CIGB-552. Res Vet Sci, 114, 502-510. doi: 10.1016/j.rvsc.2017.09.029

van de Sluis, B., Groot, A. J., Vermeulen, J., van der Wall, E., van Diest, P. J., Wijmenga, C., . . . Vooijs, M. (2009). COMMD1 Promotes pVHL and O2-Independent Proteolysis of HIF-1alpha via HSP90/70. PLoS One, 4 (10), e7332. doi: 10.1371/journal.pone.0007332

van de Sluis, B., Mao, X., Zhai, Y., Groot, A. J., Vermeulen, J. F., van der Wall, E., . . . Burstein, E. (2010). COMMD1 disrupts HIF- $1 \alpha / \beta$ dimerization and inhibits human tumor cell invasion. J Clin Invest., 
120 (6), 2119-2130. doi: doi:10.1172/JCI40583

Vonk, W. I., Wijmenga, C., Berger, R., van de Sluis, B., \& Klomp, L. W. (2010). Cu,Zn superoxide dismutase maturation and activity are regulated by COMMD1. J Biol Chem, 285 (37), 28991-29000. doi: 10.1074/jbc.M110.101477

Werle, M., Bernkop-Schnurcch. (2006). Strategies to improve plasma half life time of peptide and protein drugs. Amino acids, 30 (4), 351-367.

Wu, D., Gao, Y., Qi, Y., Chen, L., Ma, Y., \& Li, Y. (2014). Peptide-based cancer therapy: opportunity and challenge. Cancer Lett, 351 (1), 13-22. doi: 10.1016/j.canlet.2014.05.002

Yavari, B., Mahjub, R., Saidijam, M., Raigani, M., \& Soleimani, M. (2018). The Potential Use of Peptides in Cancer Treatment. Curr Protein Pept Sci, 19 (8), 759-770. doi: 10.2174/1389203719666180111150008

Yeh, D. W., Chen, Y. S., Lai, C. Y., Liu, Y. L., Lu, C. H., Lo, J. F., . . . Chuang, T. H. (2016). Downregulation of COMMD1 by miR-205 promotes a positive feedback loop for amplifying inflammatory- and stemness-associated properties of cancer cells. Cell Death Differ, 23 (5), 841-852. doi: 10.1038/cdd.2015.147.

Tables

Table 1. Prospective therapeutical applications of CIGB-552

\begin{tabular}{|c|c|c|}
\hline Disease / condition ${ }^{a}$ & Potential use & Modu \\
\hline Cancer & antineoplastic & $\mathrm{NF} x \mathrm{~B}$ \\
\hline Metastasis & antiangiogenic, antimetastatic & HIF-1 \\
\hline Colitis, IBD, CD & anti-inflammatory & $\mathrm{NF} x \mathrm{~B}$ \\
\hline Atherosclerosis & anti-lipidemic & LDL \\
\hline Cystic fibrosis & CFTR modulator & $\mathrm{CFTI}$ \\
\hline a IBD: inflammatory bowel disease; CD: Cronh's disease. & a'IBD: inflammatory bowel disease; CD: Cronh's disease. & ${ }^{\mathrm{a}} \mathrm{IBD}$ \\
\hline
\end{tabular}

\section{Figure captions}

Figure 1: Process of generation of antitumor peptide CIGB-552. The "second generation" peptide CIGB-552 was obtained by chemical modifications of L2, an antitumor peptide developed from an alanine library from CIGB-550, the LPS binding region of the LALF protein. The image illustrates the incorporation of D-aminoacids and N-terminal modifications in the peptide L2 to obtain CIGB-552. The properties acquired by each peptide are showed inside color boxes.

Figure 2: Pre-clinical summary of CIGB-552 as novel antitumor peptide. PPI: Predicted ProteinProtein Interactions.

Figure 3: Model of molecular mechanism of CIGB-552.(A) Tumor cell is characterized by inhibition of Apoptosis and NF-kB activation. COMMD1 is downregulated by proteasomal degradation. When CIGB552 penetrates the cell $(\mathbf{B})$, it interacts with COMMD1 inducing its nuclear translocation. Once in the nucleus, COMMD1 inhibits the NF-kB transcriptional activity leading eventually to a process of Apoptosis. In the cytosol the complex COMMD1-CIGB-552 activates the apoptotic pathway through oxidative stress and the modulation of Bax/Bcl-2 ratio. Red arrows indicate inhibition and green arrows indicate activation. PS: phosphatidilserine, SOD1: superoxide dismutase 1, C: cytochrome C.

Figure 4: CIGB-552 and its main metabolites. The analysis of CIGB-552 stability in serum and its characterization by ESI-MS (electrospray ionization mass spectrometry) identified the main metabolites of CIGB-552. The evaluation of in vitro properties of these metabolites, demonstrated that aminoacids of C-terminal ending are essential for antitumor activity. The properties of each metabolite are summarized in color boxes. The color scale indicates the nature of the effect. 


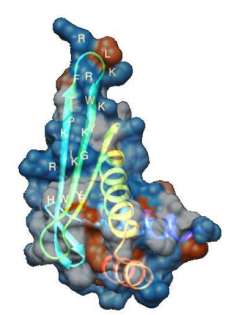

Limulus anti-LPS factor (LALF)

Antimicrobial

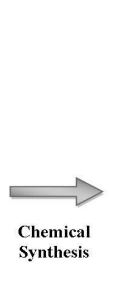

CIGB 550 (LALF $32-51$ )

HYRIKPTFRRLKWKYKGKFW

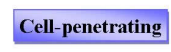

LPS-binding

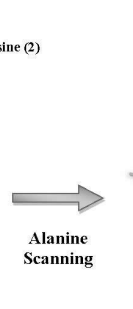

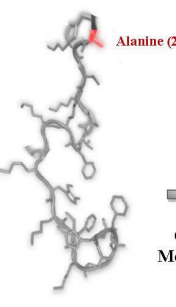

L2

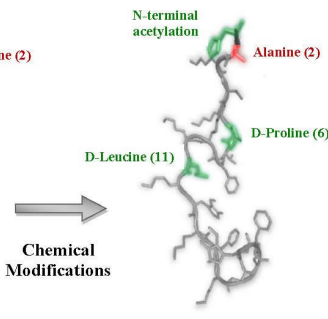

CIGB 552

Cell-penetrating

Antitumor ++

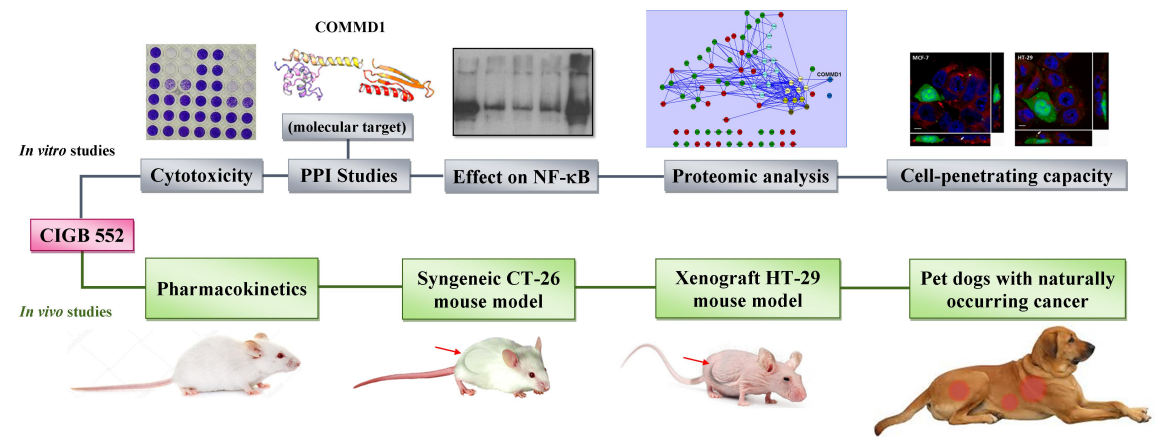



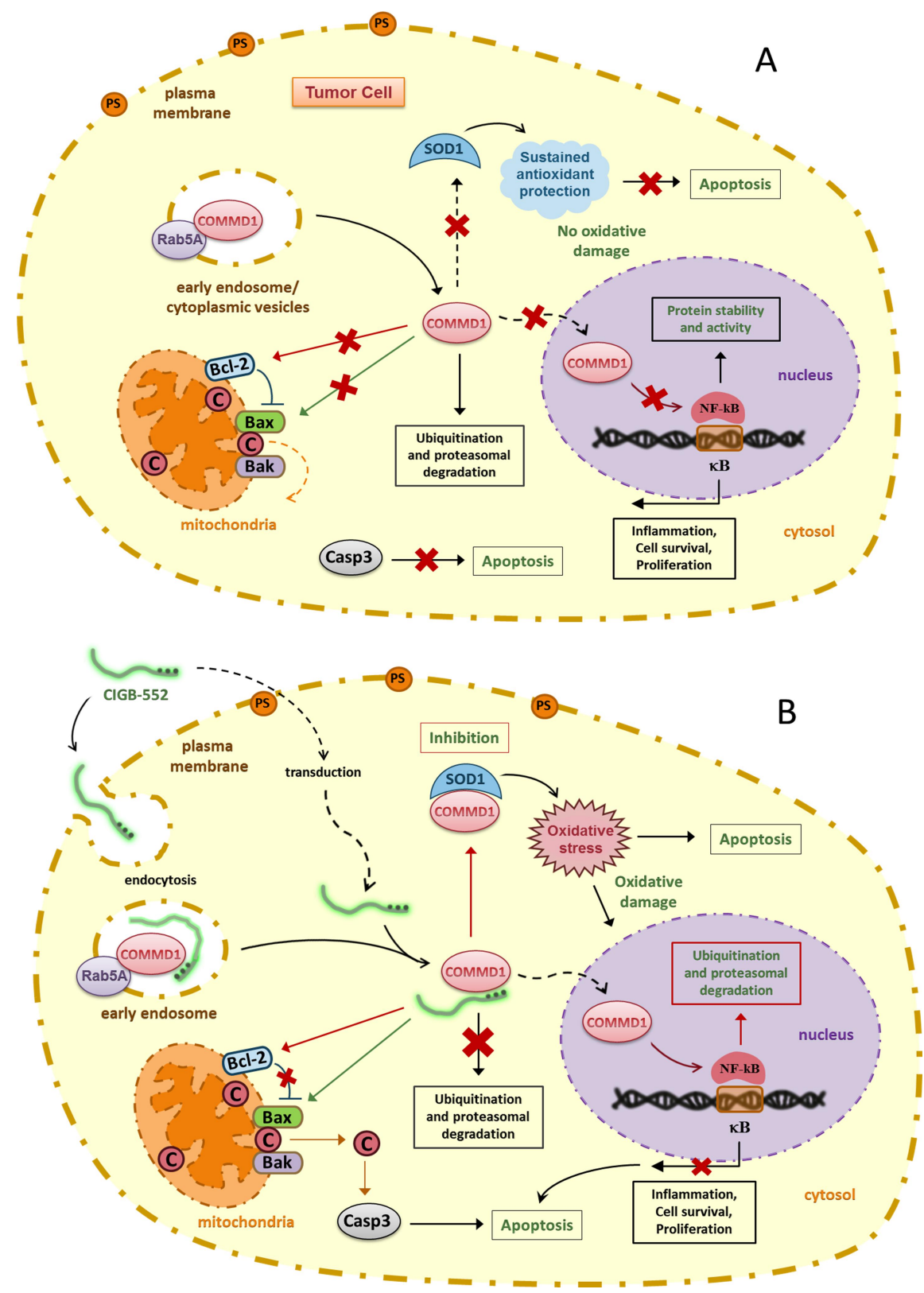


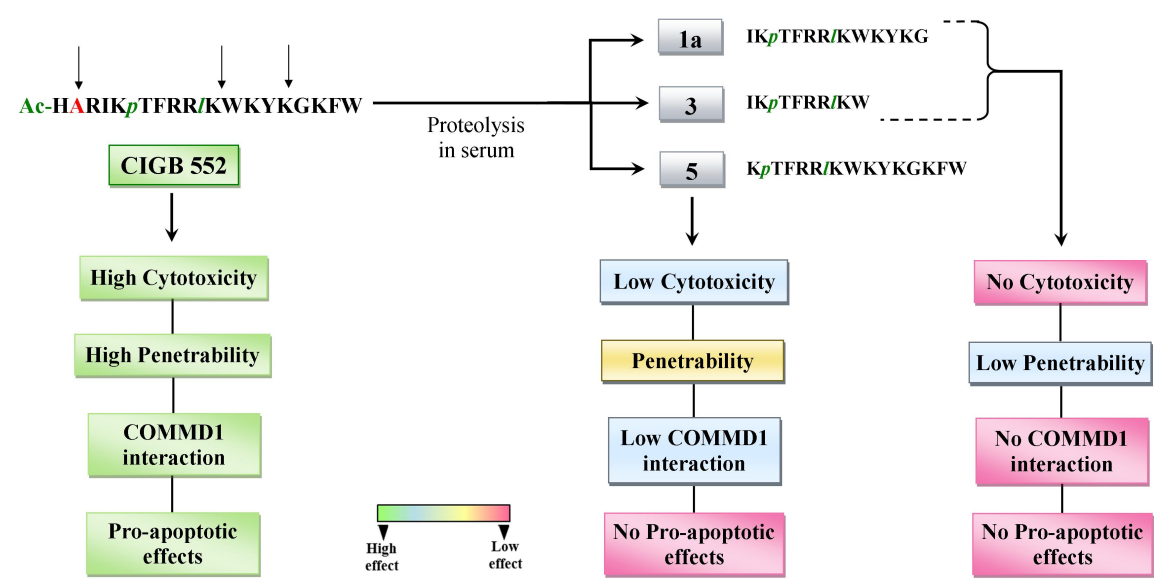

